\title{
Three- and Four-point correlators of excited bosonic twist fields
}

\section{Pascal Anastasopoulos*}

Technische Univ. Wien Inst. für Theoretische Physik, A-1040 Vienna, Austria

E-mail: pascal@hep.itp.tuwien.ac.at

\section{Mark D. Goodsell}

1- Sorbonne Universités, UPMC Univ Paris 06, UMR 7589, LPTHE, F-75005, Paris, France

2-CNRS, UMR 7589, LPTHE, F-75005, Paris, France

E-mail: goodsellalpthe.jussieu.fr

\section{Robert Richter}

II. Institut für Theoretische Physik, Hamburg University, Germany

E-mail: robert.richter@desy.de

\begin{abstract}
This proceeding is based on [1], where we computed correlation functions containing excited bosonic twist fields. Our results can be used for the phenomenological study of massive string excitations localised at D-brane intersections. Such massive states may be observable at the LHC provided that we live a universe with a low string scale.
\end{abstract}

Proceedings of the Corfu Summer Institute 2014 "School and Workshops on Elementary Particle Physics and Gravity",

3-21 September 2014

Corfu, Greece

\footnotetext{
*Speaker.
} 


\section{Introduction}

D-branes provide a very rich framework for realistic model building. One of the most exciting properties of this paradigm is that it allows for a low string scale [2-4] even at a few TeV range ${ }^{1}$. Therefore, phenomenological studies of these models are particularly interesting and several directions have been extensively analysed, such as anomalous $Z^{\prime}$ physics (see, e.g. [8-21]), Kaluza Klein states (see, e.g. [22-28]), and purely stringy signatures (see, e.g. [29-42]) ${ }^{2}$.

In this proceeding, we will take a different direction which has been less studied and focus on so-called light stringy states. More precisely, in semirealistic constructions which consist of intersecting D-branes, there is a whole tower of states living on each intersection, sharing the same quantum numbers but with masses are proportional to the intersection angle $\theta$

$$
M^{2} \sim 0, \theta M_{s}^{2}, 2 \theta M_{s}^{2}, 3 \theta M_{s}^{2}, \ldots
$$

In this framework, the Standard Model matter content is described by the first (massless) modes (for reviews see [45-48] and references therein). Such towers look like the Kaluza-Klein (KK) towers with the significant difference that in D-brane models each tower has a unique mass gap (depending on the specific angle of the intersection where each state lives) in contrast to the KK models where all particle towers share the same mass gaps (depending on the global properties of the internal space). That makes D-brane and KK models easily distinguishable.

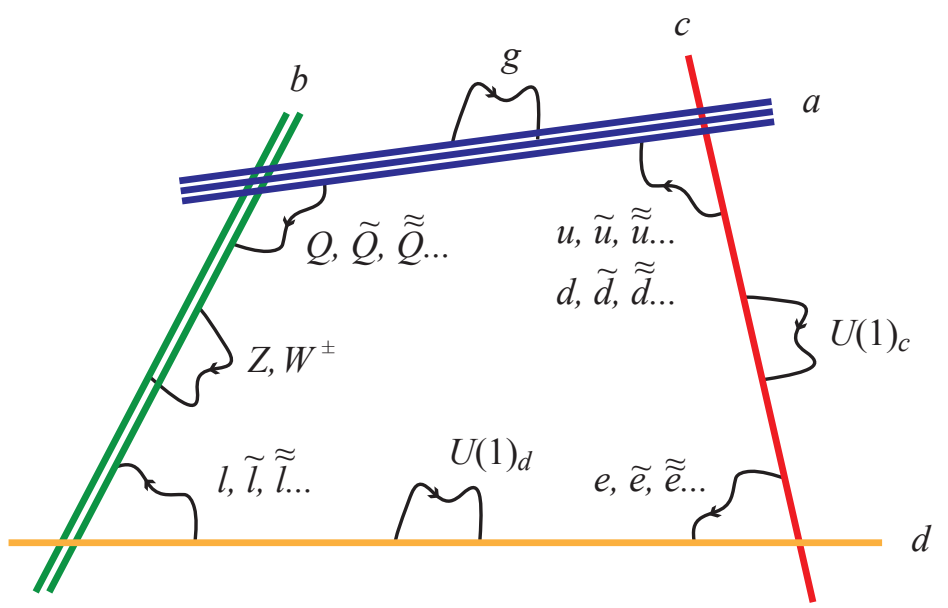

Figure 1: A D-brane realisation of the Standard Model. Gauge fields live on D-branes, matter content stretches between different D-branes. On each intersection we have a whole tower of massive copies of the corresponding Standard Model field.

If the string scale is low and some of these angles are very small, some of these excited stringy copies of the Standard Model particles become very light and can be produced at LHC. The study of decay rates and life times of these light stringy states is very interesting and it requires the knowledge of their vertex operators [49-51] as well as the relevant correlation functions ${ }^{3}$.

\footnotetext{
${ }^{1}$ At the moment, the lower bound of the string scale is at $4.5 \mathrm{TeV}$ [5-7].

${ }^{2}$ For recent reviews, see [43,44].

${ }^{3}$ For some preliminary work on excited bosonic twist field correlators, see [52-58].
} 
In [1], we evaluated various useful correlation functions involving (higher) excited bosonic twist fields, which are a crucial ingredient in the vertex operators massive stringy excitations localized at the intersections of two D-brane stacks. For simplicity and reasons of calculability we assumed the compactification manifold to be a factorizable six-torus $T^{6}=T^{2} \times T^{2} \times T^{2}$ wrapped by D6-branes (for previous related work on similar backgrounds see e.g. [59-78], for recent work, see e.g. $[79,80])$. In that case the internal part of the world sheet integrand of the scattering amplitudes splits into three separate factorizable parts for each two-tori $T^{2}$, for which one can apply the developed CFT techniques.

Summarising, we determine various correlators containing (higher) excited bosonic twist fields, where the latter live on one of three two-tori. More precisely, we derive correlators of the type

$$
\left\langle\tau_{\alpha_{1}}^{+}\left(x_{1}\right) \tilde{\tau}_{\alpha_{2}}^{+}\left(x_{2}\right) \sigma_{\alpha_{3}}^{+}\left(x_{3}\right) \ldots\right\rangle,\left\langle\omega_{\alpha_{1}}^{+}\left(x_{1}\right) \sigma_{\alpha_{2}}^{+}\left(x_{2}\right) \sigma_{\alpha_{3}}^{+}\left(x_{3}\right) \ldots\right\rangle \text {, etc }
$$

where the $\sigma$ fields are the usual bosonic twist fields while $\tau$ and $\omega$ denote the excited and double excited bosonic twist fields. The subscript denotes the intersection angle which is measured in units of $\pi$ and ranges in the interval $[0,1)$. The "+" upper-index denotes that all angles are positive.

Note that the excited twist fields $\tau$ and $\omega$ are not primary conformal fields, therefore, we first need to evaluate some higher point correlators with only primary fields, namely solely bosonic twist fields $\sigma$ as well as the conformal fields $\partial Z$ and $\partial \bar{Z}$, and next apply various operator product expansions OPE's by performing appropriate limits in order to obtain (1.2). This method is called the energy momentum tensor method and originally used in [81-83] in the context of closed string theory on orbifolds and more recently it applied to open string theory $[53,84-89]^{4}$.

This proceeding is organised as follows: In section 2 we illustrate the method which we use to evaluate correlators containing the excited bosonic twist fields. In section 3, we apply that method to compute four-point correlators with one independent angle. The appendix A contains various OPE's.

\section{The method}

Lets assume the compactification manifold to be a factorizable six-torus $T^{6}=T^{2} \times T^{2} \times T^{2}$. In terms of the compactified coordinates $Z^{i}, \bar{Z}^{i}$ we have

$$
Z^{i}=X^{2 i+2}+i X^{2 i+3}, \quad \bar{Z}^{i}=X^{2 i+2}-i X^{2 i+3}
$$

with the index $i$ denoting the three different two-tori. In the following, we will focus on a single two-torus $T^{2}$. The analysis for the other two two-tori is analogous.

In order to derive the displayed correlators (1.2) we will use the energy momentum tensor method [86-89]

- First, evaluate some extended correlators with only primary fields:

$$
\left\langle\prod_{i} \partial Z_{c l}\left(z_{i}\right) \prod_{j} \partial \bar{Z}_{c l}\left(w_{j}\right) \sigma_{\alpha}^{+}\left(x_{1}\right) \sigma_{\beta}^{+}\left(x_{2}\right) \ldots\right\rangle
$$

where it contains

\footnotetext{
${ }^{4}$ For similar work on the T-dual side with magnetized branes, see [90].
} 
1. as many $\sigma$ 's as the total number of states in the desired correlators (1.2),

2. as many $\partial Z, \partial \bar{Z}$ we need to transform some of the $\sigma$ to $\tau$ or $\omega$ 's etc using the following diagrammatic figure

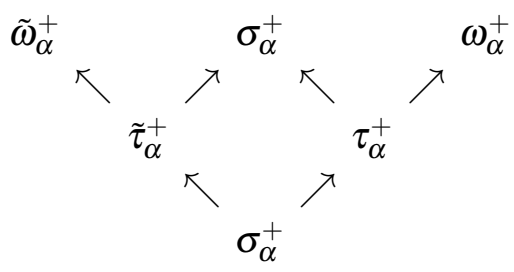

where $\nearrow, \nwarrow$ denote the OPE action of $\partial Z, \partial \bar{Z}$ on various twisted fields $\sigma, \tau, \omega$ and can be easily extended to higher excited bosonic twist fields (see appendx A).

Since $\partial Z, \partial \bar{Z}$ split to classical and quantum parts

$$
\partial Z=\partial Z_{c l}+\partial Z_{q u} \quad \partial \bar{Z}=\partial \bar{Z}_{c l}+\partial \bar{Z}_{q u}
$$

we also split the correlators to a pure quantum and some mixed parts and we evaluate them separately

- The quantum part looks like

$$
\left\langle\prod_{i} \partial Z_{q u}\left(z_{i}\right) \prod_{j} \partial \bar{Z}_{q u}\left(w_{j}\right) \sigma_{\alpha}^{+}\left(x_{1}\right) \sigma_{\beta}^{+}\left(x_{2}\right) \ldots\right\rangle
$$

we use the local behaviour

$\partial Z_{q u}(z) \partial \bar{Z}_{q u}(w) \sim \frac{1}{(z-w)^{2}}, \quad \partial Z_{q u}(z) \partial Z_{q u}(w) \sim$ regular,$\quad \partial \bar{Z}_{q u}(z) \partial \bar{Z}_{q u}(w) \sim$ regular

and the OPEs (see appendx A) in order to make an ansatz about the relevant correlators.

The above generically does not uniquely specify the correlators but we also need to impose the monodromy conditions extracted by the behaviour of $\partial Z$ and $\partial \bar{Z}$ at the specific configurations.

- The mixed parts on the other hand have the form

$$
\prod_{i^{\prime}} \partial Z_{c l}\left(\widetilde{z}_{i^{\prime}}\right) \prod_{j^{\prime}} \partial \bar{Z}_{c l}\left(\widetilde{w}_{j^{\prime}}\right)\left\langle\prod_{i^{\prime \prime}} \partial Z_{q u}\left(z_{i^{\prime \prime}}\right) \prod_{j^{\prime \prime}} \partial \bar{Z}_{q u}\left(w_{j^{\prime \prime}}\right) \sigma_{\alpha}^{+}\left(x_{1}\right) \sigma_{\beta}^{+}\left(x_{2}\right) \ldots\right\rangle
$$

with $\sum_{i^{\prime}} 1+\sum_{i^{\prime \prime}} 1=\sum_{i} 1$ and $\sum_{j^{\prime}} 1+\sum_{j^{\prime \prime}} 1=\sum_{j} 1$. Therefore, it splits into a product of $\partial Z_{c l}$ and $\partial \bar{Z}_{c l}$ which are known for the specific configurations $[87,88]$ and a pure quantum part, similar to (2.5) which can be independently evaluated by the energy momentum tensor method described above.

Finally, any correlator is suppressed by the world-sheet instanton, which is given by $e^{-S_{c l}}$ where the whole world-sheet instanton contribution ${ }^{5}$

$$
S_{c l}=\frac{1}{4 \pi \alpha^{\prime}} \int d^{2} z\left\{\partial Z_{c l}(z) \partial Z_{c l}(z)+\partial \bar{Z}_{c l}(z) \partial \bar{Z}_{c l}(z)\right\}
$$

\footnotetext{
${ }^{5}$ Note that we applied the doubling trick that extends the world-sheet from the upper half complex plane to the whole complex plane.
} 
is a sum over all possible closed triangles connecting the three intersection points [86-89] $(\text { See also [32] })^{6}$.

- Having at hand the extended correlator (2.2), we take various limits $z_{i} \rightarrow x_{k}, w_{j} \rightarrow x_{m}$ (and also $\left.\widetilde{z}_{i} \rightarrow x_{l}, \widetilde{w}_{j} \rightarrow x_{n}\right)$ which brings $\partial Z\left(z_{i}\right)$ and $\partial \bar{Z}\left(w_{j}\right)$ close to the position of $\sigma\left(x_{k}\right)$ and we can use the OPEs given in appendix A in order to determine the correlators (1.2).

In the next chapter we will provide one example of the method above and we will evaluate the correlator function of four twisted fields with two excited $\tau, \tilde{\tau}(\operatorname{aka}\langle\tau \tilde{\tau} \sigma \sigma\rangle)$ or one double excited field $\omega$ (aka $\langle\omega \sigma \sigma \sigma\rangle$ ) with one independent angle. The complete list of our results is provided in [1].

\section{Four-point correlators containing two excited twist fields}

As mentioned above, in this section we will evaluate four-point correlation function containing two exited twist fields $\tau, \tilde{\tau}$ or one double-excited twist field $\omega$

$$
\begin{aligned}
& \left\langle\tau_{\alpha}^{+}\left(x_{1}\right) \tilde{\tau}_{\alpha}^{-}\left(x_{2}\right) \sigma_{\alpha}^{+}\left(x_{3}\right) \sigma_{\alpha}^{-}\left(x_{4}\right)\right\rangle \\
& \left\langle\tau_{\alpha}^{+}\left(x_{1}\right) \sigma_{\alpha}^{-}\left(x_{2}\right) \tilde{\tau}_{\alpha}^{+}\left(x_{3}\right) \sigma_{\alpha}^{-}\left(x_{4}\right)\right\rangle \\
& \left\langle\tau_{\alpha}^{+}\left(x_{1}\right) \tau_{\alpha}^{-}\left(x_{2}\right) \sigma_{\alpha}^{+}\left(x_{3}\right) \sigma_{\alpha}^{-}\left(x_{4}\right)\right\rangle \\
& \left\langle\tau_{\alpha}^{+}\left(x_{1}\right) \sigma_{\alpha}^{-}\left(x_{2}\right) \tau_{\alpha}^{+}\left(x_{3}\right) \sigma_{\alpha}^{-}\left(x_{4}\right)\right\rangle \\
& \left\langle\omega_{\alpha}^{+}\left(x_{1}\right) \sigma_{\alpha}^{-}\left(x_{2}\right) \sigma_{\alpha}^{+}\left(x_{3}\right) \sigma_{\alpha}^{-}\left(x_{4}\right)\right\rangle
\end{aligned}
$$

for one independant angle $\alpha$, where the boundary conditions on the world-sheet are

$$
\begin{aligned}
& \partial Z-\partial \bar{Z}=0 \text { for }\left(-\infty, x_{1}\right) \cup\left(x_{2}, x_{3}\right) \cup\left(x_{4}, \infty\right) \\
& e^{i \pi \alpha} \partial Z-e^{-i \pi \alpha} \partial \bar{Z}=0 \text { for }\left(x_{1}, x_{2}\right) \cup\left(x_{3}, x_{4}\right) .
\end{aligned}
$$

Following the steps which were presented above

- first, we derive the extended six-point correlators

$$
\begin{aligned}
& \left\langle\partial Z(z) \partial \bar{Z}(w) \sigma_{\alpha}^{+}\left(x_{1}\right) \sigma_{\alpha}^{-}\left(x_{2}\right) \sigma_{\alpha}^{+}\left(x_{3}\right) \sigma_{\alpha}^{-}\left(x_{4}\right)\right\rangle \\
& \left\langle\partial \bar{Z}(z) \partial \bar{Z}(w) \sigma_{\alpha}^{+}\left(x_{1}\right) \sigma_{\alpha}^{-}\left(x_{2}\right) \sigma_{\alpha}^{+}\left(x_{3}\right) \sigma_{\alpha}^{-}\left(x_{4}\right)\right\rangle \\
& \left\langle\partial Z(z) \partial Z(w) \sigma_{\alpha}^{+}\left(x_{1}\right) \sigma_{\alpha}^{-}\left(x_{2}\right) \sigma_{\alpha}^{+}\left(x_{3}\right) \sigma_{\alpha}^{-}\left(x_{4}\right)\right\rangle .
\end{aligned}
$$

which split to a pure quantum and a mixed part (where $\partial Z(z), \partial \bar{Z}(w)$ are classical) and we will deal with them separately.

- the quantum part

\footnotetext{
${ }^{6}$ For analogous results in the T-dual IIB framework with magnetized D-branes, see [90,91].
} 
Let us define the following auxiliary functions

$$
\begin{aligned}
g(z, w) & =\frac{\left\langle\partial Z_{q u}(z) \partial \bar{Z}_{q u}(w) \sigma_{\alpha}^{+}\left(x_{1}\right) \sigma_{\alpha}^{-}\left(x_{2}\right) \sigma_{\alpha}^{+}\left(x_{3}\right) \sigma_{\alpha}^{-}\left(x_{4}\right)\right\rangle}{\left\langle\sigma_{\alpha}^{+}\left(x_{1}\right) \sigma_{\alpha}^{-}\left(x_{2}\right) \sigma_{\alpha}^{+}\left(x_{3}\right) \sigma_{\alpha}^{-}\left(x_{4}\right)\right\rangle} \\
k(z, w) & =\frac{\left\langle\partial \bar{Z}_{q u}(z) \partial \bar{Z}_{q u}(w) \sigma_{\alpha}^{+}\left(x_{1}\right) \sigma_{\alpha}^{-}\left(x_{2}\right) \sigma_{\alpha}^{+}\left(x_{3}\right) \sigma_{\alpha}^{-}\left(x_{4}\right)\right\rangle}{\left\langle\sigma_{\alpha}^{+}\left(x_{1}\right) \sigma_{\alpha}^{-}\left(x_{2}\right) \sigma_{\alpha}^{+}\left(x_{3}\right) \sigma_{\alpha}^{-}\left(x_{4}\right)\right\rangle} \\
m(z, w) & =\frac{\left\langle\partial Z_{q u}(z) \partial Z_{q u}(w) \sigma_{\alpha}^{+}\left(x_{1}\right) \sigma_{\alpha}^{-}\left(x_{2}\right) \sigma_{\alpha}^{+}\left(x_{3}\right) \sigma_{\alpha}^{-}\left(x_{4}\right)\right\rangle}{\left\langle\sigma_{\alpha}^{+}\left(x_{1}\right) \sigma_{\alpha}^{-}\left(x_{2}\right) \sigma_{\alpha}^{+}\left(x_{3}\right) \sigma_{\alpha}^{-}\left(x_{4}\right)\right\rangle}
\end{aligned}
$$

where the four-point correlator is given by $[53,84-88,92]$

$$
\begin{aligned}
& \left\langle\sigma_{\alpha}^{+}(0) \sigma_{\alpha}^{-}(x) \sigma_{\alpha}^{+}(1) \sigma_{\alpha}^{-}(\infty)\right\rangle \\
& \quad=\frac{\sin ^{\frac{1}{2}}(\pi \alpha)}{{ }_{2} F_{1}\left[\alpha, 1-\alpha, 1, \frac{x_{12} x_{34}}{x_{13} x_{24}}\right]^{\frac{1}{2}}{ }_{2} F_{1}\left[\alpha, 1-\alpha, 1, \frac{x_{14} x_{23}}{x_{13} x_{24}}\right]^{\frac{1}{2}}}\left[\frac{x_{12} x_{23} x_{14} x_{34}}{x_{13} x_{24}}\right]
\end{aligned}
$$

with the hypergeometric function

$$
{ }_{2} F_{1}[\alpha, \beta, \gamma, x]=\frac{\Gamma(\gamma)}{\Gamma(\alpha) \Gamma(\beta)} \int_{0}^{1} d z z^{\beta-1}(1-z)^{\gamma-\beta-1}(1-x z)^{-\alpha}
$$

and $\Gamma(\alpha)$ being $\Gamma(\alpha)=\int_{0}^{\infty} d z z^{\alpha-1} e^{-z}$.

Using the local behaviour of the bosonic twist fields (A.1) as well as (2.6) we obtain for the functions $g(z, w), k(z, w)$ and $m(z, w)$

$$
\begin{aligned}
& g(z, w)=\alpha^{\prime} \omega_{1-\alpha, 1-\alpha}(z) \omega_{\alpha, \alpha}(w)\left\{\frac{P}{(z-w)^{2}}+A\left(\left\{x_{i}\right\}\right)\right\} \\
& k(z, w)=\alpha^{\prime} \omega_{\alpha, \alpha}(z) \omega_{\alpha, \alpha}(w) B\left(\left\{x_{i}\right\}\right) \\
& m(z, w)=\alpha^{\prime} \omega_{1-\alpha, 1-\alpha}(z) \omega_{1-\alpha, 1-\alpha}(w) C\left(\left\{x_{i}\right\}\right)
\end{aligned}
$$

where we use various symmetries of the functions under the exchanges of the $x_{i}$ 's. Here

$$
\omega_{\alpha, \beta}(z)=\left(z-x_{1}\right)^{-\alpha}\left(z-x_{2}\right)^{-1+\alpha}\left(z-x_{3}\right)^{-\beta}\left(z-x_{4}\right)^{-1+\beta} .
$$

and $P$ takes the form

$$
\begin{aligned}
2 P= & (1-\xi)\left(z-x_{1}\right)\left(z-x_{2}\right)\left(w-x_{3}\right)\left(w-x_{4}\right) \\
& +2(-\alpha+\xi)\left(z-x_{1}\right)\left(w-x_{2}\right)\left(z-x_{3}\right)\left(w-x_{4}\right) \\
& +(1-\xi)\left(z-x_{1}\right)\left(w-x_{2}\right)\left(w-x_{3}\right)\left(z-x_{4}\right) \\
& +(1-\xi)\left(w-x_{1}\right)\left(z-x_{2}\right)\left(z-x_{3}\right)\left(w-x_{4}\right) \\
& +2(-1+\alpha+\xi)\left(w-x_{1}\right)\left(z-x_{2}\right)\left(w-x_{3}\right)\left(z-x_{4}\right) \\
& +(1-\xi)\left(w-x_{1}\right)\left(w-x_{2}\right)\left(z-x_{3}\right)\left(z-x_{4}\right) .
\end{aligned}
$$

The $\xi$ is a free parameter that is neither fixed by the local behavior nor by any symmetry of the functions. The $A\left(\left\{x_{i}\right\}\right), B\left(\left\{x_{i}\right\}\right)$ and $C\left(\left\{x_{i}\right\}\right)$ are functions on positions of the bosonic twist fields only and thus independent of $z$ and $w$. Those functions will be 
determined using constraints arising from global monodromies of the boundary conditions $(3.6)^{7}$

$$
\begin{aligned}
& \int_{x}^{1} d z(g(z, w)-k(z, w))=0, \quad \int_{0}^{x} d z\left(e^{i \pi \alpha} g(z, w)-e^{-i \pi \alpha} k(z, w)\right)=0 \\
& \int_{x}^{1} d w(m(z, w)-g(z, w))=0, \quad \int_{0}^{x} d w\left(e^{i \pi \alpha} m(z, w)-e^{-i \pi \alpha} g(z, w)\right)=0
\end{aligned}
$$

After using $S L(2, \mathbf{R})$ symmetry to fix $x_{1}=0, x_{2}=x, x_{3}=1$ and $x_{4}=x_{\infty}$ and solving the above conditions we get

$$
\begin{aligned}
& \left\langle\partial Z_{q u}(z) \partial \bar{Z}_{q u}(w) \sigma_{\alpha}^{+}(0) \sigma_{\alpha}^{-}(x) \sigma_{\alpha}^{+}(1) \sigma_{\alpha}^{-}(\infty)\right\rangle \\
& =\alpha^{\prime} \frac{\sqrt{\pi}[x(1-x)]^{-\alpha(1-\alpha)}[z(w-x)(z-1)]^{\alpha-1}[w(z-x)(w-1)]^{-\alpha}}{\sqrt{\Gamma(\alpha) \Gamma(1-\alpha){ }_{2} F_{1}[1-\alpha, \alpha, 1, x]_{2} F_{1}[1-\alpha, \alpha, 1,1-x]}} \\
& \times\left\{(1-\alpha) \frac{z(w-x)(z-1)}{(z-w)^{2}}+\alpha \frac{w(z-x)(w-1)}{(z-w)^{2}}\right. \\
& \left.+\frac{1}{2} \alpha(1-\alpha)\left(x \frac{{ }_{2} F_{1}[1-\alpha, \alpha, 2, x]}{{ }_{2} F_{1}[1-\alpha, \alpha, 1, x]}-(1-x) \frac{{ }_{2} F_{1}[1-\alpha, \alpha, 2,1-x]}{{ }_{2} F_{1}[1-\alpha, \alpha, 1,1-x]}\right)\right\} . \\
& \left\langle\partial \bar{Z}_{q u}(z) \partial \bar{Z}_{q u}(w) \sigma_{\alpha}^{+}(0) \sigma_{\alpha}^{-}(x) \sigma_{\alpha}^{+}(1) \sigma_{\alpha}^{-}(\infty)\right\rangle \\
& =\alpha^{\prime} \alpha e^{2 i \pi \alpha} \frac{\sqrt{\pi}[x(1-x)]^{-\alpha(1-\alpha)}[z(z-1) w(w-1)]^{-\alpha}[(z-x)(w-x)]^{\alpha-1}}{2 \sqrt{\Gamma(\alpha) \Gamma(1-\alpha)_{2} F_{1}[1-\alpha, \alpha, 1, x]_{2} F_{1}[1-\alpha, \alpha, 1,1-x]}} \\
& \times\left\{1-\frac{{ }_{2} F_{1}[-\alpha, \alpha, 1,1-x]}{{ }_{2} F_{1}[1-\alpha, \alpha, 1,1-x]}-\frac{{ }_{2} F_{1}[-\alpha, \alpha, 1, x]}{{ }_{2} F_{1}[1-\alpha, \alpha, 1, x]}\right\} \text {. } \\
& \left\langle\partial Z_{q u}(z) \partial Z_{q u}(w) \sigma_{\alpha}^{+}(0) \sigma_{\alpha}^{-}(x) \sigma_{\alpha}^{+}(1) \sigma_{\alpha}^{-}(\infty)\right\rangle \\
& =\alpha^{\prime}(1-\alpha) e^{-2 \pi i \alpha} \frac{\sqrt{\pi}[x(1-x)]^{-\alpha(1-\alpha)}[z(z-1) w(w-1)]^{-\alpha}[(z-x)(w-x)]^{\alpha-1}}{2 \sqrt{\Gamma(\alpha) \Gamma(1-\alpha)_{2} F_{1}[1-\alpha, \alpha, 1, x]_{2} F_{1}[1-\alpha, \alpha, 1,1-x]}} \\
& \times\left\{1-\frac{{ }_{2} F_{1}[1-\alpha, \alpha-1,1,1-x]}{{ }_{2} F_{1}[1-\alpha, \alpha, 1,1-x]}-\frac{{ }_{2} F_{1}[1-\alpha, \alpha-1,1, x]}{{ }_{2} F_{1}[1-\alpha, \alpha, 1, x]}\right\} \text {. }
\end{aligned}
$$

Note that all results are as expected completely independent of $\xi$.

- The mixed parts are given by

$$
\begin{aligned}
& \partial Z_{c l}(z) \partial \bar{Z}_{c l}(w)\left\langle\sigma_{\alpha}^{+}\left(x_{1}\right) \sigma_{\alpha}^{-}\left(x_{2}\right) \sigma_{\alpha}^{+}\left(x_{3}\right) \sigma_{\alpha}^{-}\left(x_{4}\right)\right\rangle \\
& \partial \bar{Z}_{c l}(z) \partial \bar{Z}_{c l}(w)\left\langle\sigma_{\alpha}^{+}\left(x_{1}\right) \sigma_{\alpha}^{-}\left(x_{2}\right) \sigma_{\alpha}^{+}\left(x_{3}\right) \sigma_{\alpha}^{-}\left(x_{4}\right)\right\rangle \\
& \partial Z_{c l}(z) \partial Z_{c l}(w)\left\langle\sigma_{\alpha}^{+}\left(x_{1}\right) \sigma_{\alpha}^{-}\left(x_{2}\right) \sigma_{\alpha}^{+}\left(x_{3}\right) \sigma_{\alpha}^{-}\left(x_{4}\right)\right\rangle .
\end{aligned}
$$

since mixed parts with one quantum $\partial Z_{q u}$ or $\partial \bar{Z}_{q u}$ vanish. Now, it is very simple to evaluate (3.23) since all pieces are known. The four point correlator is given in (3.11).

\footnotetext{
${ }^{7}$ The setup with four bosonic twist field insertions has two independent world-sheet contours which we choose without loss of generality to be 0 to $x$ and $x$ to 1 .
} 
The asymptotic behaviour close to the insertion of the bosonic twist fields specify the classical solutions $[86,88]^{8}$

$$
\begin{aligned}
& \partial Z_{c l}(z)=-\sqrt{\alpha^{\prime}} \frac{\sin (\pi \alpha)}{2 \pi} \frac{v_{b}[m]+e^{i \pi \alpha} v_{a}[n] \tau(x)}{{ }_{2} F_{1}[\alpha, 1-\alpha, 1,1-x]} \omega_{1-\alpha, 1-\alpha}(z) \\
& \partial \bar{Z}_{c l}(z)=-\sqrt{\alpha^{\prime}} \frac{\sin (\pi \alpha)}{2 \pi} \frac{v_{b}[m]-e^{i \pi \alpha} v_{a}[n] \tau(x)}{{ }_{2} F_{1}[\alpha, 1-\alpha, 1,1-x]} \omega_{\alpha, \alpha}(z)
\end{aligned}
$$

where $\tau(x)$ is given by the ratio

$$
\tau(x)=\frac{{ }_{2} F_{1}[\alpha, 1-\alpha, 1,1-x]}{{ }_{2} F_{1}[\alpha, 1-\alpha, 1, x]}
$$

and

$$
v_{a}[n]=f_{3}-f_{1}+n \widetilde{L}_{a}, \quad v_{b}[m]=f_{3}-f_{2}+m \widetilde{L}_{b}
$$

with $n, m$ being integers and $\widetilde{L}_{x}$ given by

$$
\widetilde{L}_{a}=\frac{\left|I_{b c}\right|}{\operatorname{gcd}\left(\left|I_{a b}\right|,\left|I_{b c}\right|, \mid I_{c a}\right) \mid} L_{a} .
$$

In eq. (3.27) $I_{x y}=n_{x} m_{y}-n_{y} m_{x}$ is the intersection number between two D-brane stacks $x$ and $y, L_{x}=R_{1} \sqrt{n_{x}^{2}+\left(m_{x} \mathscr{T}\right)^{2}}$ the length of the D-brane stack $x$, with $\left(n_{x}, m_{x}\right)$ being the wrapping numbers and $\mathscr{T}$ denoting the complex structure of the two-torus. In the simple setup in which all three D-brane stacks intersect each other exactly once $\widetilde{L}_{x}$ coincides with the length $L_{x}$ of the respective D-brane.

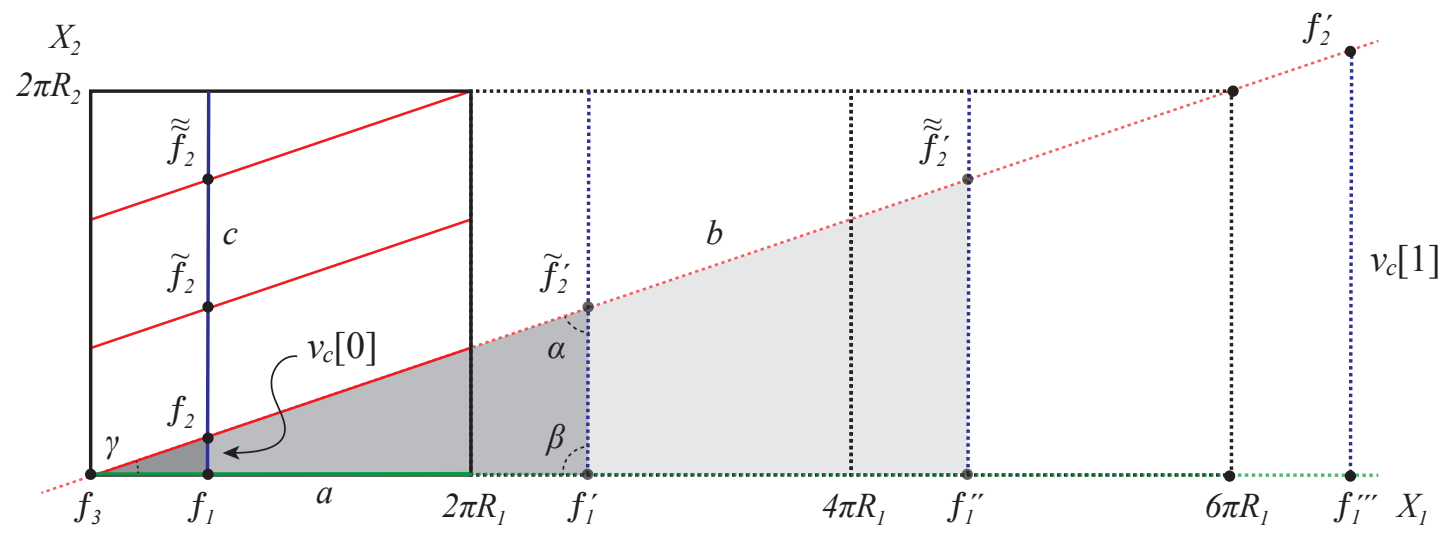

Figure 2: Let us consider the three intersecting D-brane stacks wrapping the one-cycles $a=(1,0), b=(3,1)$, $c=(0,1)$ on a rectangular two-torus.

Let us examplify the notion of the $v_{c}[n]$ with the explicit example depicted in figure 2 . Here we have three stacks of D-branes, $a, b$ and $c$, wrapping one-cycles on a $T^{2}$. Their

\footnotetext{
${ }^{8}$ Here we used $S L(2, \mathbf{R})$ invariance to fix the twist field insertions to $x_{1}=0, x_{2}=x, x_{3}=1$ and $x_{4}=x_{\infty}=\infty$. Furthermore we suppress all $x_{\infty}$ dependence.
} 
intersection numbers are given by $I_{a b}=1, I_{b c}=3, I_{a c}=1$, where $f_{1}$ denotes the intersection between D-brane stack $c$ and $a$ while $f_{2}, \widetilde{f}_{2}$ and $\widetilde{\widetilde{f}}_{2}$ denote the three intersection points among the D-brane stacks $b$ and $c$. The primed $f$ 's are the corresponding lattice shifted intersection points. For this explicit example the vectors $v_{c}[0]$ and $v_{c}[1]$ take the form $v_{c}[0]=f_{1}-f_{2}$ and $v_{c}[1]=f_{1}^{\prime \prime \prime}-f_{2}^{\prime}=f_{1}-f_{2}+\widetilde{L}_{c}$, respectively.

Combining the quantum and mix parts we obtain the extended six-point correlators:

$$
\begin{gathered}
\left\langle\partial Z(z) \partial \bar{Z}(w) \sigma_{\alpha}^{+}(0) \sigma_{\alpha}^{-}(x) \sigma_{\alpha}^{+}(1) \sigma_{\alpha}^{-}(\infty)\right\rangle \\
=\alpha^{\prime} \frac{\sqrt{\pi}[x(1-x)]^{-\alpha(1-\alpha)}[z(w-x)(z-1)]^{\alpha-1}[w(z-x)(w-1)]^{-\alpha}}{\sqrt{\Gamma(\alpha) \Gamma(1-\alpha){ }_{2} F_{1}[1-\alpha, \alpha, 1, x]{ }_{2} F_{1}[1-\alpha, \alpha, 1,1-x]}} \\
\times \sum_{n, m}\left\{(1-\alpha) \frac{z(w-x)(z-1)}{(z-w)^{2}}+\alpha \frac{w(z-x)(w-1)}{(z-w)^{2}}-\frac{\sin ^{2}(\pi \alpha)}{4 \pi^{2}} \frac{v_{b}^{2}[m]-e^{2 i \pi \alpha} v_{a}^{2}[n] \tau^{2}(x)}{{ }_{2} F_{1}[1-\alpha, \alpha, 1,1-x]^{2}}\right. \\
\left.\quad+\frac{1}{2} \alpha(1-\alpha)\left(x \frac{{ }_{2} F_{1}[1-\alpha, \alpha, 2, x]}{{ }_{2} F_{1}[1-\alpha, \alpha, 1, x]}-(1-x) \frac{{ }_{2} F_{1}[1-\alpha, \alpha, 2,1-x]}{{ }_{2} F_{1}[1-\alpha, \alpha, 1,1-x]}\right)\right\} e^{-S_{c l}[n, m]} \\
\left\langle\partial \bar{Z}(z) \partial \bar{Z}(w) \sigma_{\alpha}^{+}(0) \sigma_{\alpha}^{-}(x) \sigma_{\alpha}^{+}(1) \sigma_{\alpha}^{-}(\infty)\right\rangle \\
=\alpha^{\prime} \alpha e^{2 i \pi \alpha} \frac{\sqrt{\pi}[x(1-x)]^{-\alpha(1-\alpha)}[z(z-1) w(w-1)]^{-\alpha}[(z-x)(w-x)]^{\alpha-1}}{2 \sqrt{\Gamma(\alpha) \Gamma(1-\alpha){ }_{2} F_{1}[1-\alpha, \alpha, 1, x]{ }_{2} F_{1}[1-\alpha, \alpha, 1,1-x]}} \\
\quad \times \sum_{n, m}\left\{1-\frac{{ }_{2} F_{1}[-\alpha, \alpha, 1,1-x]}{{ }_{2} F_{1}[1-\alpha, \alpha, 1,1-x]}-\frac{{ }_{2} F_{1}[-\alpha, \alpha, 1, x]}{{ }_{2} F_{1}[1-\alpha, \alpha, 1, x]}\right. \\
\quad \sin ^{2}(\pi \alpha) \\
2 \pi^{2} \alpha
\end{gathered}
$$

$$
\begin{array}{r}
\left\langle\partial Z(z) \partial Z(w) \sigma_{\alpha}^{+}(0) \sigma_{\alpha}^{-}(x) \sigma_{\alpha}^{+}(1) \sigma_{\alpha}^{-}(\infty)\right\rangle \\
=\alpha^{\prime}(1-\alpha) e^{-2 \pi i \alpha} \frac{\sqrt{\pi}[x(1-x)]^{-\alpha(1-\alpha)}[z(z-1) w(w-1)]^{\alpha-1}[(z-x)(w-x)]^{-\alpha}}{2 \sqrt{\Gamma(\alpha) \Gamma(1-\alpha){ }_{2} F_{1}[1-\alpha, \alpha, 1, x]{ }_{2} F_{1}[1-\alpha, \alpha, 1,1-x]}} \\
\times \sum_{n, m}\left\{1-\frac{{ }_{2} F_{1}[1-\alpha, \alpha-1,1,1-x]}{{ }_{2} F_{1}[1-\alpha, \alpha, 1,1-x]}-\frac{{ }_{2} F_{1}[1-\alpha, \alpha-1,1, x]}{{ }_{2} F_{1}[1-\alpha, \alpha, 1, x]}\right. \\
\left.+\frac{\sin ^{2}(\pi \alpha)}{2 \pi^{2}(1-\alpha)} \frac{\left(v_{b}[m]+e^{i \pi \alpha} v_{a}[n] \tau(x)\right)^{2}}{{ }_{2} F_{1}[\alpha, 1-\alpha, 1,1-x]^{2}}\right\} e^{-S_{c l}[n, m]}
\end{array}
$$

Note that all correlators are suppressed by world-sheet instanton contributions which can be easily computed applying (2.8) as well as using the classical solutions (3.24). One obtains

$$
S_{c l}^{T^{2}}[n, m]=\pi \sin (\pi a)\left\{\left|v_{a}[n]\right|^{2} \tau(x)+\left|v_{b}[m]\right|^{2} \tau(1-x)\right\},
$$

where $\tau(x)$ is given by (3.25).

- With the extended six-point correlators at hand, we can derive the various four-point correlators containing higher excited twist fields by taking the appropriate limits. 
Taking for example the limit $z \rightarrow 0$ and $w \rightarrow x$ of $\left\langle\partial Z \partial \bar{Z} \sigma_{\alpha}^{+} \sigma_{\alpha}^{-} \sigma_{\alpha}^{+} \sigma_{\alpha}^{-}\right\rangle$(3.28) and using the OPE's (A.1) one finds

$$
\begin{aligned}
& \left\langle\tau_{\alpha}^{+}(0) \tilde{\tau}_{\alpha}^{-}(x) \sigma_{\alpha}^{+}(1) \sigma_{\alpha}^{-}(\infty)\right\rangle \\
& =\alpha^{\prime} \frac{\sqrt{\pi} x^{-\alpha(3-\alpha)}(1-x)^{-\alpha(2-\alpha)}}{\sqrt{\Gamma(\alpha) \Gamma(1-\alpha){ }_{2} F_{1}[\alpha, 1-\alpha, 1, x]_{2} F_{1}[\alpha, 1-\alpha, 1,1-x]}} \\
& \quad \times \sum_{n, m}\left\{\alpha(1-x)+\frac{1}{2} \alpha(1-\alpha)\left(x \frac{{ }_{2} F_{1}[1-\alpha, \alpha, 2, x]}{{ }_{2} F_{1}[1-\alpha, \alpha, 1, x]}-(1-x) \frac{{ }_{2} F_{1}[1-\alpha, \alpha, 2,1-x]}{{ }_{2} F_{1}[1-\alpha, \alpha, 1,1-x]}\right)\right. \\
& \left.\quad-\frac{\sin ^{2}(\pi \alpha)}{4 \pi^{2}} \frac{v_{b}^{2}[m]-e^{2 i \pi \alpha} v_{a}^{2}[n] \tau^{2}(x)}{{ }_{2} F_{1}[1-\alpha, \alpha, 1,1-x]^{2}}\right\} e^{-S_{c l}[n, m]}
\end{aligned}
$$

which after reinstating all $x_{i}$ dependence gives ${ }^{9}$

$$
\begin{aligned}
& \left\langle\tau_{\alpha}^{+}\left(x_{1}\right) \tilde{\tau}_{\alpha}^{-}\left(x_{2}\right) \sigma_{\alpha}^{+}\left(x_{3}\right) \sigma_{\alpha}^{-}\left(x_{4}\right)\right\rangle \\
& =\alpha^{\prime} \frac{\sqrt{\pi} x_{12}^{-\alpha(3-\alpha)} x_{34}^{-\alpha(1-\alpha)}\left(\frac{x_{13} x_{24}}{x_{14} x_{23}}\right)^{\alpha(2-\alpha)}}{\sqrt{\Gamma(\alpha) \Gamma(1-\alpha){ }_{2} F_{1}[\alpha, 1-\alpha, 1, x]_{2} F_{1}[\alpha, 1-\alpha, 1,1-x]}} \\
& \quad \times \sum_{n, m}\left\{\alpha(1-x)+\frac{1}{2} \alpha(1-\alpha)\left(x \frac{{ }_{2} F_{1}[1-\alpha, \alpha, 2, x]}{{ }_{2} F_{1}[1-\alpha, \alpha, 1, x]}-(1-x) \frac{{ }_{2} F_{1}[1-\alpha, \alpha, 2,1-x]}{{ }_{2} F_{1}[1-\alpha, \alpha, 1,1-x]}\right)\right. \\
& \left.-\frac{\sin ^{2}(\pi \alpha)}{4 \pi^{2}} \frac{v_{b}^{2}[m]-e^{2 i \pi \alpha} v_{a}^{2}[n] \tau^{2}(x)}{{ }_{2} F_{1}[1-\alpha, \alpha, 1,1-x]^{2}}\right\} e^{-S_{c l}[n, m]} .
\end{aligned}
$$

The above technique can be applied to any of the six-point correlators generating various four-point correlators.

$$
\begin{gathered}
\left\langle\partial \mathrm{Z} \overline{\mathrm{Z}} \sigma_{\alpha}^{+} \sigma_{\alpha}^{-} \sigma_{\alpha}^{+} \sigma_{\alpha}^{-}\right\rangle \rightarrow\left\{\begin{array}{l}
\left\langle\tau_{\alpha}^{+}\left(x_{1}\right) \widetilde{\tau}_{\alpha}^{-}\left(x_{2}\right) \sigma_{\alpha}^{+}\left(x_{3}\right) \sigma_{\alpha}^{-}\left(x_{4}\right)\right\rangle \\
\left\langle\tau_{\alpha}^{+}\left(x_{1}\right) \sigma_{\alpha}^{-}\left(x_{2}\right) \tilde{\tau}_{\alpha}^{+}\left(x_{3}\right) \sigma_{\alpha}^{-}\left(x_{4}\right)\right\rangle
\end{array}\right. \\
\left\langle\partial Z \partial Z \sigma_{\alpha}^{+} \sigma_{\alpha}^{-} \sigma_{\alpha}^{+} \sigma_{\alpha}^{-}\right\rangle \rightarrow\left\{\begin{array}{l}
\left\langle\tau_{\alpha}^{+}\left(x_{1}\right) \tau_{\alpha}^{-}\left(x_{2}\right) \sigma_{\alpha}^{+}\left(x_{3}\right) \sigma_{\alpha}^{-}\left(x_{4}\right)\right\rangle \\
\left\langle\tau_{\alpha}^{+}\left(x_{1}\right) \sigma_{\alpha}^{-}\left(x_{2}\right) \tau_{\alpha}^{+}\left(x_{3}\right) \sigma_{\alpha}^{-}\left(x_{4}\right)\right\rangle \\
\left\langle\omega_{\alpha}^{+}\left(x_{1}\right) \sigma_{\alpha}^{-}\left(x_{2}\right) \sigma_{\alpha}^{+}\left(x_{3}\right) \sigma_{\alpha}^{-}\left(x_{4}\right)\right\rangle
\end{array}\right. \\
\left\langle\partial \bar{Z} \partial \bar{Z} \sigma_{\alpha}^{+} \sigma_{\alpha}^{-} \sigma_{\alpha}^{+} \sigma_{\alpha}^{-}\right\rangle \rightarrow\left\{\begin{array}{l}
\left\langle\widetilde{\tau}_{\alpha}^{+}\left(x_{1}\right) \tilde{\tau}_{\alpha}^{-}\left(x_{2}\right) \sigma_{\alpha}^{+}\left(x_{3}\right) \sigma_{\alpha}^{-}\left(x_{4}\right)\right\rangle \\
\left\langle\widetilde{\tau}_{\alpha}^{+}\left(x_{1}\right) \sigma_{\alpha}^{-}\left(x_{2}\right) \widetilde{\tau}_{\alpha}^{+}\left(x_{3}\right) \sigma_{\alpha}^{-}\left(x_{4}\right)\right\rangle \\
\left\langle\widetilde{\omega}_{\alpha}^{+}\left(x_{1}\right) \sigma_{\alpha}^{-}\left(x_{2}\right) \sigma_{\alpha}^{+}\left(x_{3}\right) \sigma_{\alpha}^{-}\left(x_{4}\right)\right\rangle
\end{array}\right.
\end{gathered}
$$

Note that all other correlators (like $\left\langle\widetilde{\tau}_{\alpha}^{+} \tau_{\alpha}^{-} \sigma_{\alpha}^{+} \sigma_{\alpha}^{-}\right\rangle$) can be obtained by the ones presented above by simply applying the rules displayed in (A.2)

$$
\begin{array}{lll}
\sigma_{\alpha}^{-}=\sigma_{1-\alpha}^{+} & \tau_{\alpha}^{-}=\tau_{1-\alpha}^{+} & \omega_{\alpha}^{-}=\omega_{1-\alpha}^{+} \\
\widetilde{\tau}_{\alpha}^{-}=\widetilde{\tau}_{1-\alpha}^{+} & \widetilde{\omega}_{\alpha}^{-}=\widetilde{\omega}_{1-\alpha}^{+} .
\end{array}
$$

${ }^{9}$ Here we use the fact that an open string four-point correlator is given by

$$
\left\langle\phi_{h_{1}}\left(x_{1}\right) \phi_{h_{2}}\left(x_{2}\right) \phi_{h_{3}}\left(x_{3}\right) \phi_{h_{4}}\left(x_{4}\right)\right\rangle=\mathscr{F}(x) \prod_{i<j} x_{i j}^{-\left(h_{i}+h_{j}\right)+\frac{\Delta}{3}},
$$

where $\Delta=\sum_{i=1}^{4} h_{i}$ and $x=\frac{x_{12} x_{34}}{x_{13} x_{24}}$. This expression allows us to reinstate the $x_{i}$ dependence in all the four-point correlators. 
All our results for one and two independent angles can be found in [1].

\section{Conclusions}

In this proceeding we have presented a method for the evaluation of three- and four-point correlators containing excited bosonic twist fields. The knowledge of these correlators is required for the evaluation of lifetime and decay rates of massive string excitations that arise at intersections of D-branes.

In order to evaluate these correlators, we took a detour and determined higher-point correlators containing the conformal fields $\partial Z$ and $\partial \bar{Z}$ as well as the regular bosonic twist fields $\sigma$. Given those correlators we performed various limits to derive the three- and four-point correlators containing higher excited bosonic twist fields.

Our results can be used in order to evaluate decay rates and lifetimes for the massive excited stringy states of the Standard Model matter fields [93]. If the string scale is low (at a few $\mathrm{TeV}$ range) and the intersection angles between the D-branes very small, then such light stringy states can be the first stringy effect to be observed at LHC.

\section{Acknowledgements}

We would like to thank the organizers of Corfu 2013 for giving us the opportunity to present this work. P. A. is supported by the Austrian Science Fund (FWF) program P 26731-N27. M. D. G. was supported during the time the work upon which this proceeding is based was originally performed by a Marie Curie Intra European Fellowship within the 7th European Community Framework Programme and by ERC advanced grant 226371. R. R. acknowledges the support from the FP7 Marie Curie Actions of the European Commission, via the Intra-European Fellowships (project number: 328170).

\section{A. OPE's of twisted states}

Here, we provide the OPEs of $\partial Z(x)$ and $\partial \bar{Z}(x)$ on twisted fields:

$$
\begin{aligned}
\partial Z(z) \sigma_{\alpha}^{+}(w) & \sim(z-w)^{\alpha-1} \tau_{\alpha}^{+}(w) & & \partial \bar{Z}(z) \sigma_{\alpha}^{+}(w) \sim(z-w)^{-\alpha} \widetilde{\tau}_{\alpha}^{+}(w) \\
\partial Z(z) \tau_{\alpha}^{+}(w) & \sim(z-w)^{\alpha-1} \omega_{\alpha}^{+}(w) & & \partial \bar{Z}(z) \tau_{\alpha}^{+}(w) \sim(z-w)^{-\alpha-1} \sigma_{\alpha}^{+}(w) \\
\partial Z(z) \widetilde{\tau}_{\alpha}^{+}(w) & \sim(z-w)^{-2+\alpha} \sigma_{\alpha}^{+}(w) & & \partial \bar{Z}(z) \omega_{\alpha}^{+}(w) \sim(z-w)^{-\alpha-1} \tau_{\alpha}^{+}(w) \\
\partial Z(z) \sigma_{\alpha}^{-}(w) & \sim(z-w)^{-\alpha} \tau_{\alpha}^{-}(w) & & \partial \bar{Z}(z) \widetilde{\tau}_{\alpha}^{+}(w) \sim(z-w)^{-\alpha} \widetilde{\omega}_{\alpha}^{+}(w) \\
\partial Z(z) \tau_{\alpha}^{-}(w) & \sim(z-w)^{-\alpha} \omega_{\alpha}^{-}(w) & & \partial \bar{Z}(z) \sigma_{\alpha}^{-}(w) \sim(z-w)^{\alpha-1} \widetilde{\tau}_{\alpha}^{-}(w) \\
\partial Z(z) \widetilde{\tau}_{\alpha}^{-}(w) & \sim(z-w)^{-1+\alpha} \sigma_{\alpha}^{-}(w) & & \partial \bar{Z}(z) \tau_{\alpha}^{-}(w) \sim(z-w)^{-2+\alpha} \sigma_{\alpha}^{-}(w) \\
\partial Z(z) \widetilde{\omega}_{\alpha}^{-}(w) & \sim(z-w)^{-1+\alpha} \widetilde{\tau}_{\alpha}^{-}(w) & & \partial \bar{Z}(z) \widetilde{\tau}_{\alpha}^{-}(w) \sim(z-w)^{-1-\alpha} \widetilde{\omega}_{\alpha}^{-}(w)
\end{aligned}
$$

The above OPEs suggest the following identifications among twist- and anti-twist fields

$$
\sigma_{\alpha}^{-}(z)=\sigma_{1-\alpha}^{+}(z) \quad \tau_{\alpha}^{-}(z)=\tau_{1-\alpha}^{+}(z) \quad \tilde{\tau}_{\alpha}^{-}(z)=\widetilde{\tau}_{1-\alpha}^{+}(z)
$$

which can be easily generalised to higher excited twist fields. 


\section{References}

[1] P. Anastasopoulos, M. D. Goodsell, and R. Richter, Three- and Four-point correlators of excited bosonic twist fields, JHEP 1310 (2013) 182, [arXiv: 1305. 7166].

[2] N. Arkani-Hamed, S. Dimopoulos, and G. Dvali, The Hierarchy problem and new dimensions at a millimeter, Phys.Lett. B429 (1998) 263-272, [hep-ph / 9803315$].$

[3] I. Antoniadis, S. Dimopoulos, and G. Dvali, Millimeter range forces in superstring theories with weak scale compactification, Nucl.Phys. B516 (1998) 70-82, [hep-ph/9710204].

[4] I. Antoniadis, N. Arkani-Hamed, S. Dimopoulos, and G. Dvali, New dimensions at a millimeter to a Fermi and superstrings at a TeV, Phys.Lett. B436 (1998) 257-263, [hep-ph/ 9804398 ].

[5] CMS Collaboration Collaboration, S. Chatrchyan et. al., Search for Resonances in the Dijet Mass Spectrum from 7 TeV pp Collisions at CMS, Phys.Lett. B704 (2011) 123-142, [arXiv:1107.4771].

[6] ATLAS Collaboration Collaboration, G. Aad et. al., ATLAS search for new phenomena in dijet mass and angular distributions using pp collisions at $\sqrt{s}=7 \mathrm{TeV}$, JHEP 1301 (2013) 029, [arXiv:1210.1718].

[7] CMS Collaboration Collaboration, S. Chatrchyan et. al., Search for narrow resonances using the dijet mass spectrum in pp collisions at $\sqrt{s}=8$ ??TeV, Phys.Rev. D87 (2013), no. 11114015 , [arXiv:1302.4794].

[8] E. Kiritsis and P. Anastasopoulos, The Anomalous magnetic moment of the muon in the D-brane realization of the standard model, JHEP 0205 (2002) 054, [hep-ph/ 02012 95].

[9] I. Antoniadis, E. Kiritsis, and J. Rizos, Anomalous U(1)s in type 1 superstring vacua, Nucl.Phys. B637 (2002) 92-118, [hep-th/ 0204153$].$

[10] D. Ghilencea, L. Ibáñez, N. Irges, and F. Quevedo, TeV scale Z-prime bosons from D-branes, JHEP $\mathbf{0 2 0 8}$ (2002) 016, [hep-ph/ 0205083$].$

[11] P. Anastasopoulos, 4-D anomalous U(1)'s, their masses and their relation to 6-D anomalies, JHEP $\mathbf{0 3 0 8}$ (2003) 005, [hep-th/ 0306042$].$

[12] P. Anastasopoulos, Anomalous U(1)s masses in nonsupersymmetric open string vacua, Phys.Lett. B588 (2004) 119-126, [hep-th/ 0402105$].$

[13] P. Burikham, T. Figy, and T. Han, TeV-scale string resonances at hadron colliders, Phys.Rev. D71 (2005) 016005, [hep-ph/0 411094 ].

[14] C. Corianò, N. Irges, and E. Kiritsis, On the effective theory of low scale orientifold string vacua, Nucl.Phys. B746 (2006) 77-135, [hep-ph/ 0510332$].$

[15] P. Anastasopoulos, M. Bianchi, E. Dudas, and E. Kiritsis, Anomalies, anomalous U(1)'s and generalized Chern-Simons terms, JHEP 0611 (2006) 057, [hep-th / 060522 5].

[16] P. Anastasopoulos, F. Fucito, A. Lionetto, G. Pradisi, A. Racioppi, and Y. Stanev, Minimal Anomalous U(1)-prime Extension of the MSSM, Phys.Rev. D78 (2008) 085014, [arXiv: 0804.1156 ].

[17] R. Armillis, C. Coriano, M. Guzzi, and S. Morelli, An Anomalous Extra Z Prime from Intersecting Branes with Drell-Yan and Direct Photons at the LHC, Nucl.Phys. B814 (2009) 156-179, [arXiv:0809.3772]. 
[18] F. Fucito, A. Lionetto, A. Mammarella, and A. Racioppi, Stueckelino dark matter in anomalous U(1)-prime models, Eur.Phys.J. C69 (2010) 455-465, [arXiv : 0811.1953$].$

[19] L. A. Anchordoqui, H. Goldberg, X. Huang, D. Lüst, and T. R. Taylor, Stringy origin of Tevatron Wjj anomaly, Phys.Lett. B701 (2011) 224-228, [arXiv: 1104.2302 ].

[20] L. A. Anchordoqui, I. Antoniadis, H. Goldberg, X. Huang, D. Lüst, and T. Taylor, Z'-gauge Bosons as Harbingers of Low Mass Strings, Phys.Rev. D85 (2012) 086003, [arXiv: 1107 . 4309].

[21] L. A. Anchordoqui, I. Antoniadis, H. Goldberg, X. Huang, D. Lüst, T. Taylor, and B. Vlcek, LHC Phenomenology and Cosmology of String-Inspired Intersecting D-Brane Models, Phys.Rev. D86 (2012) 066004, [arXiv:1206.2537].

[22] E. Dudas and J. Mourad, String theory predictions for future accelerators, Nucl.Phys. $\mathbf{5 5 7 5}$ (2000) 3-34, [hep-th/9911019].

[23] E. Accomando, I. Antoniadis, and K. Benakli, Looking for TeV scale strings and extra dimensions, Nucl.Phys. B579 (2000) 3-16, [hep-ph/9912287].

[24] S. Cullen, M. Perelstein, and M. E. Peskin, TeV strings and collider probes of large extra dimensions, Phys.Rev. D62 (2000) 055012, [hep-ph/ 0001166 ].

[25] C. Burgess, J. Matias, and F. Quevedo, MSLED: A Minimal supersymmetric large extra dimensions scenario, Nucl.Phys. B706 (2005) 71-99, [hep-ph / 0404135$].$

[26] D. Chialva, R. Iengo, and J. G. Russo, Cross sections for production of closed superstrings at high energy colliders in brane world models, Phys.Rev. D71 (2005) 106009, [hep-ph/0 03125 ].

[27] M. Cicoli, C. Burgess, and F. Quevedo, Anisotropic Modulus Stabilisation: Strings at LHC Scales with Micron-sized Extra Dimensions, JHEP 1110 (2011) 119, [arXiv: 1105.2107$].$

[28] D. Chialva, P. B. Dev, and A. Mazumdar, Multiple dark matter scenarios from ubiquitous stringy throats, Phys.Rev. D87 (2013), no. 6 063522, [arXiv:1211.0250].

[29] M. Bianchi and A. V. Santini, String predictions for near future colliders from one-loop scattering amplitudes around D-brane worlds, JHEP 0612 (2006) 010, [hep-th/ 0607224 ].

[30] L. A. Anchordoqui, H. Goldberg, S. Nawata, and T. R. Taylor, Jet signals for low mass strings at the LHC, Phys.Rev.Lett. 100 (2008) 171603, [arXiv:0712.0386].

[31] L. A. Anchordoqui, H. Goldberg, S. Nawata, and T. R. Taylor, Direct photons as probes of low mass strings at the CERN LHC, Phys.Rev. D78 (2008) 016005, [arXiv: 0804 . 2013].

[32] D. Lüst, S. Stieberger, and T. R. Taylor, The LHC String Hunter's Companion, Nucl.Phys. B808 (2009) 1-52, [arXiv:0807.3333].

[33] L. A. Anchordoqui, H. Goldberg, D. Lüst, S. Nawata, S. Stieberger, and T. Taylor, Dijet signals for low mass strings at the LHC, Phys.Rev.Lett. 101 (2008) 241803, [arXiv: 0808 . 0497].

[34] L. A. Anchordoqui, H. Goldberg, D. Lüst, S. Stieberger, and T. R. Taylor, String Phenomenology at the LHC, Mod.Phys.Lett. A24 (2009) 2481-2490, [arXiv: 0909.2216 ].

[35] D. Lüst, O. Schlotterer, S. Stieberger, and T. Taylor, The LHC String Hunter's Companion (II): Five-Particle Amplitudes and Universal Properties, Nucl.Phys. $\mathbf{B 8 2 8}$ (2010) 139-200, [arXiv:0908.0409].

[36] L. A. Anchordoqui, H. Goldberg, D. Lüst, S. Nawata, S. Stieberger, and T. Taylor, LHC Phenomenology for String Hunters, Nucl.Phys. B821 (2009) 181-196, [arXiv: 0904.3547 ]. 
[37] L. A. Anchordoqui, W.-Z. Feng, H. Goldberg, X. Huang, and T. R. Taylor, Searching for string resonances in $e^{+} e^{-}$and $\gamma \gamma$ collisions, Phys.Rev. D83 (2011) 106006, [arXiv: 1012.3466 ].

[38] W.-Z. Feng, D. Lüst, O. Schlotterer, S. Stieberger, and T. R. Taylor, Direct Production of Lightest Regge Resonances, Nucl.Phys. B843 (2011) 570-601, [arXiv: 1007.5254 ].

[39] Z. Dong, T. Han, M.-x. Huang, and G. Shiu, Top Quarks as a Window to String Resonances, JHEP 1009 (2010) 048, [arXiv: 1004.5441$].$

[40] D. Carmi, TeV Scale Strings and Scattering Amplitudes at the LHC, arXiv: 1109.5161.

[41] M. Hashi and N. Kitazawa, Detectability of the second resonance of low-scale string models at the LHC, JHEP 1303 (2013) 127, [arXiv: 1212 . 5372].

[42] L. A. Anchordoqui, I. Antoniadis, D.-C. Dai, W.-Z. Feng, H. Goldberg, et. al., String Resonances at Hadron Colliders, arXiv:1407.8120.

[43] D. Lüst and T. R. Taylor, Limits on Stringy Signals at the LHC, arXiv:1308.1619.

[44] D. Berenstein, TeV-Scale strings, arXiv:1401.4491.

[45] R. Blumenhagen, M. Cvetič, P. Langacker, and G. Shiu, Toward realistic intersecting D-brane models, Ann.Rev.Nucl.Part.Sci. 55 (2005) 71-139, [hep-th/ 0502005 ].

[46] R. Blumenhagen, B. Körs, D. Lüst, and S. Stieberger, Four-dimensional String Compactifications with D-Branes, Orientifolds and Fluxes, Phys.Rept. 445 (2007) 1-193, [hep-th/ 0610327 ].

[47] F. Marchesano, Progress in D-brane model building, Fortsch.Phys. 55 (2007) 491-518, [hep-th/0702094].

[48] M. Cvetič and J. Halverson, TASI Lectures: Particle Physics from Perturbative and Non-perturbative Effects in D-braneworlds, arXiv:1101.2907.

[49] P. Anastasopoulos, M. Bianchi, and R. Richter, Light stringy states, JHEP 1203 (2012) 068, [arXiv:1110.5424].

[50] J. P. Conlon and L. T. Witkowski, Scattering and Sequestering of Blow-Up Moduli in Local String Models, JHEP 1112 (2011) 028, [arXiv:1109. 4153].

[51] G. Honecker, Kaehler metrics and gauge kinetic functions for intersecting D6-branes on toroidal orbifolds - The complete perturbative story, Fortsch.Phys. 60 (2012) 243-326, [arXiv:1109.3192].

[52] E. Kiritsis and C. Kounnas, String propagation in gravitational wave backgrounds, Phys.Lett. $\mathbf{B 3 2 0}$ (1994) 264-272, [hep-th/9310202].

[53] J. R. David, Tachyon condensation using the disc partition function, JHEP 07 (2001) 009, [hep-th/0012089].

[54] D. Lüst, P. Mayr, R. Richter, and S. Stieberger, Scattering of gauge, matter, and moduli fields from intersecting branes, Nucl.Phys. B696 (2004) 205-250, [hep-th / 0404134 ].

[55] G. D’Appollonio and E. Kiritsis, String interactions in gravitational wave backgrounds, Nucl.Phys. B674 (2003) 80-170, [hep-th/ 0305081$]$.

[56] G. D'Appollonio and E. Kiritsis, D-branes and BCFT in Hpp-wave backgrounds, Nucl.Phys. B712 (2005) 433-512, [hep-th/ 0410269$].$

[57] I. Pesando, The generating function of amplitudes with $N$ twisted and $M$ untwisted states, arXiv:1107.5525. 
[58] I. Pesando, Green functions and twist correlators for $N$ branes at angles, Nucl.Phys. $\mathbf{B 8 6 6}$ (2013) 87-123, [arXiv:1206.1431].

[59] R. Blumenhagen, L. Görlich, B. Körs, and D. Lüst, Noncommutative compactifications of type I strings on tori with magnetic background flux, JHEP 0010 (2000) 006, [hep-th/ 0007024 ].

[60] C. Angelantonj, I. Antoniadis, E. Dudas, and A. Sagnotti, Type I strings on magnetized orbifolds and brane transmutation, Phys.Lett. B489 (2000) 223-232, [hep-th/ 0007090 ].

[61] G. Aldazabal, S. Franco, L. E. Ibáñez, R. Rabadán, and A. Uranga, Intersecting brane worlds, JHEP 0102 (2001) 047, [hep-ph/ 0011132$].$

[62] G. Aldazabal, S. Franco, L. E. Ibáñez, R. Rabadán, and A. Uranga, D = 4 chiral string compactifications from intersecting branes, J.Math.Phys. 42 (2001) 3103-3126, [hep-th/0011073].

[63] S. Förste, G. Honecker, and R. Schreyer, Supersymmetric $Z(N) \times Z(M)$ orientifolds in 4-D with D branes at angles, Nucl.Phys. B593 (2001) 127-154, [hep-th/ 0008250$].$

[64] L. E. Ibáñez, F. Marchesano, and R. Rabádan, Getting just the standard model at intersecting branes, JHEP 0111 (2001) 002, [hep-th/ 0105155 ].

[65] M. Cvetič, G. Shiu, and A. M. Uranga, Three family supersymmetric standard - like models from intersecting brane worlds, Phys.Rev.Lett. 87 (2001) 201801, [hep-th/ 0107143 ].

[66] M. Cvetič, G. Shiu, and A. M. Uranga, Chiral four-dimensional N=1 supersymmetric type $2 A$ orientifolds from intersecting D6 branes, Nucl.Phys. B615 (2001) 3-32, [hep-th/ 0107166 ].

[67] G. Honecker, Chiral supersymmetric models on an orientifold of $Z(4) \times Z(2)$ with intersecting D6-branes, Nucl.Phys. B666 (2003) 175-196, [hep-th/ 0303015$].$

[68] M. Cvetič, P. Langacker, T.-j. Li, and T. Liu, D6-brane splitting on type IIA orientifolds, Nucl.Phys. B709 (2005) 241-266, [hep-th/ 0407178$].$

[69] G. Honecker and T. Ott, Getting just the supersymmetric standard model at intersecting branes on the Z(6) orientifold, Phys.Rev. D70 (2004) 126010, [hep-th/ 0404055 ].

[70] R. Blumenhagen, F. Gmeiner, G. Honecker, D. Lüst, and T. Weigand, The Statistics of supersymmetric D-brane models, Nucl.Phys. B713 (2005) 83-135, [hep-th/ 0411173$].$

[71] R. Blumenhagen, M. Cvetič, F. Marchesano, and G. Shiu, Chiral D-brane models with frozen open string moduli, JHEP 0503 (2005) 050, [hep-th/ 05020 95].

[72] F. Gmeiner, R. Blumenhagen, G. Honecker, D. Lüst, and T. Weigand, One in a billion: MSSM-like D-brane statistics, JHEP 0601 (2006) 004, [hep-th/ 0510170$].$

[73] D. Bailin and A. Love, Towards the supersymmetric standard model from intersecting D6-branes on the Z-prime(6) orientifold, Nucl.Phys. B755 (2006) 79-111, [hep-th/ 0603172$].$

[74] M. Cvetič and P. Langacker, New Grand Unified Models with Intersecting D6-branes, Neutrino Masses, and Flipped SU(5), Nucl.Phys. B776 (2007) 118-137, [hep-th/ 0607238 ].

[75] C.-M. Chen, T. Li, V. Mayes, and D. V. Nanopoulos, A Realistic world from intersecting D6-branes, Phys.Lett. B665 (2008) 267-270, [hep-th / 0703280$].$

[76] D. Bailin and A. Love, Almost the supersymmetric standard model from intersecting D6-branes on the Z(6)-prime orientifold, Phys.Lett. B651 (2007) 324-328, [arXiv: 0705.0646 ]. 
[77] D. Bailin and A. Love, Constructing the supersymmetric Standard Model from intersecting D6-branes on the Z(6)-prime orientifold, Nucl.Phys. B809 (2009) 64-109, [arXiv: 0801 . 3385].

[78] F. Gmeiner and G. Honecker, Millions of Standard Models on Z-prime(6)?, JHEP 0807 (2008) 052, [arXiv:0806.3039].

[79] G. Honecker, M. Ripka, and W. Staessens, The Importance of Being Rigid: D6-Brane Model Building on $T^{6} / Z_{2} x Z_{6}^{\prime}$ with Discrete Torsion, Nucl.Phys. $\mathbf{B 8 6 8}$ (2013) 156-222, [arXiv: 1209.3010 ].

[80] G. Honecker and J. Vanhoof, Yukawa couplings and masses of non-chiral states for the Standard Model on D6-branes on T6/Z6', JHEP 1204 (2012) 085, [arXiv: 1201.3604 ].

[81] S. Hamidi and C. Vafa, Interactions on Orbifolds, Nucl.Phys. B279 (1987) 465.

[82] L. J. Dixon, D. Friedan, E. J. Martinec, and S. H. Shenker, The Conformal Field Theory of Orbifolds, Nucl.Phys. B282 (1987) 13-73.

[83] T. Burwick, R. Kaiser, and H. Muller, General Yukawa couplings of strings on Z(N) orbifolds, Nucl.Phys. B355 (1991) 689-711.

[84] E. Gava, K. S. Narain, and M. H. Sarmadi, On the bound states of p-and (p+2)-branes, Nucl. Phys. B504 (1997) 214-238, [hep-th/ 9704006$].$

[85] J. R. David, Tachyon condensation in the D0/D4 system, JHEP 10 (2000) 004, [hep-th/ 0007235$].$

[86] M. Cvetič and I. Papadimitriou, Conformal field theory couplings for intersecting D-branes on orientifolds, Phys.Rev. D68 (2003) 046001, [hep-th/ 0303083 ].

[87] S. Abel and A. Owen, Interactions in intersecting brane models, Nucl.Phys. B663 (2003) 197-214, [hep-th/0303124].

[88] S. Abel and A. Owen, N point amplitudes in intersecting brane models, Nucl.Phys. $\mathbf{6 6 8 2}$ (2004) 183-216, [hep-th/0310257].

[89] D. Cremades, L. Ibáñez, and F. Marchesano, Yukawa couplings in intersecting D-brane models, JHEP 0307 (2003) 038, [hep-th/ 0302105$].$

[90] D. Cremades, L. Ibáñez, and F. Marchesano, Computing Yukawa couplings from magnetized extra dimensions, JHEP 0405 (2004) 079, [hep-th / 040422 9].

[91] I. Antoniadis, A. Kumar, and B. Panda, Fermion Wavefunctions in Magnetized branes: Theta identities and Yukawa couplings, Nucl.Phys. B823 (2009) 116-173, [arXiv: 0904.0910$].$

[92] P. Anastasopoulos, M. Bianchi, and R. Richter, On closed-string twist-field correlators and their open-string descendants, arXiv:1110.5359.

[93] P. Anastasopoulos and R. Richter, Production of light stringy states, arXiv: 1408.4810. 